
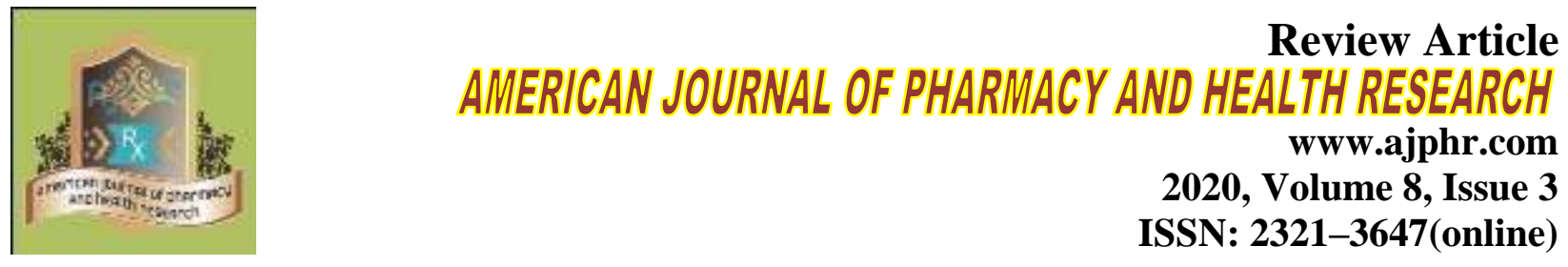

\title{
A Short Review On Benzimidazole and Their Derivatives
}

Gayatri D. Patil, Aaditya R. Nikam, Niranjan B. Karanke, Azam Z. Shaikh Department of Pharmaceutical chemistry, Ahinsa Institute of pharmacy Dhule road Dondaicha425408.

\section{ABSTRACT}

Benzimidazole derivatives are versatile nitrogen containing heterocyclic compound which have long been known as a promising class of biologically active compounds possessing wide variety of a biologically active compound like antiprotozoal, anticoagulant, antifungal, antihistaminic, antiulcer activities. Benzimidazole is outstanding effective compounds and these are a number of reviews available for biochemical and pharmacological studies. This review article covers the most active benzimidazole derivative and discusses the structure and their uses.

Keyword: Benzimidazole, Heterocyclic compound, Benzimidazole derivative, Antifungal, Antiprotozoal activity, Antihistaminic 


\section{INTRODUCTION}

The entire heterocyclic compound has great interest in pharmaceutical chemistry. Out of this heterocyclic compound the be fused heterocyclic compound i.e. benzimidazole and its derivatives have wide variety of biological activities, in addition to that the benzimidazole have played very important role in the development of theory in heterocyclic chemistry and also extensively in organic synthesis. ${ }^{[1]}$

Benzimidazole is produced by condensation of o-phenylenedamine with formic acid or the equivalent trimethyl orthoformate:

\section{$\mathrm{C}_{6} \mathrm{H}_{4}\left(\mathrm{NH}_{2}\right)_{2}+\mathrm{HC}\left(\mathrm{OCH}_{3}\right)_{3} \rightarrow \mathrm{C}_{6} \mathrm{H}_{4} \mathrm{~N}(\mathrm{NH}) \mathrm{CH}+3 \mathrm{CH}_{3} \mathrm{OH}$}

2-Subtituted derivatives are obtain when the condensation is conducted with aldehyde in the place of formic acid, followed by oxidation. This method affords 2-Benzimidazoles.

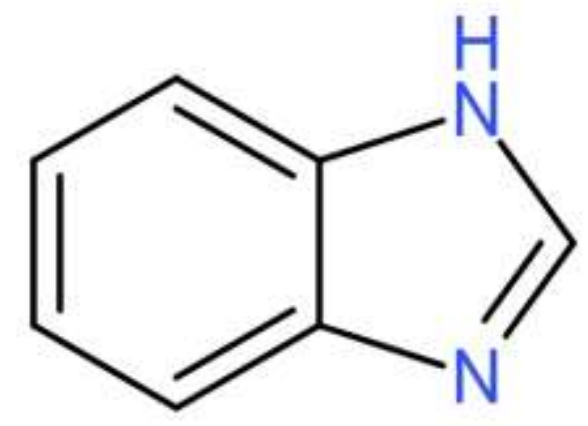

- IUPAC NAME: - 1H-1,3-Benzimidazole

- Chemical formula: $\mathrm{C}_{7} \mathrm{H}_{6} \mathrm{~N}_{2}$

- $\quad$ Molecular mass: 118.139 g.mol ${ }^{-1}$

- $\quad$ Melting point: 170 to $172{ }^{\circ} \mathrm{c}[338 \text { to } 342 \mathrm{f}]^{[2]}$

Study of structural and modifications and their pharmacological action:

The use of benzimidazole dated many years back. ${ }^{[3]}$ In 1990 various benzimidazole derivatives were derivatives synthesized with substitution of fluorine, propylene, tetrahydroquinoline and cyclized compound which resulted in compound with increased stability, bioavailability and significant biological activity, ${ }^{[4][5]}$ it was show that the substitution on pyridine by electron donating group and substitution with long chain of propyl, acetamido, thio, thiazole-amino, tetra methyl piperidine on pyridine resulting in good antiulcer activity. ${ }^{[6][7]}$ 
<smiles>CN1CC(C(=O)O)CC1=O</smiles>
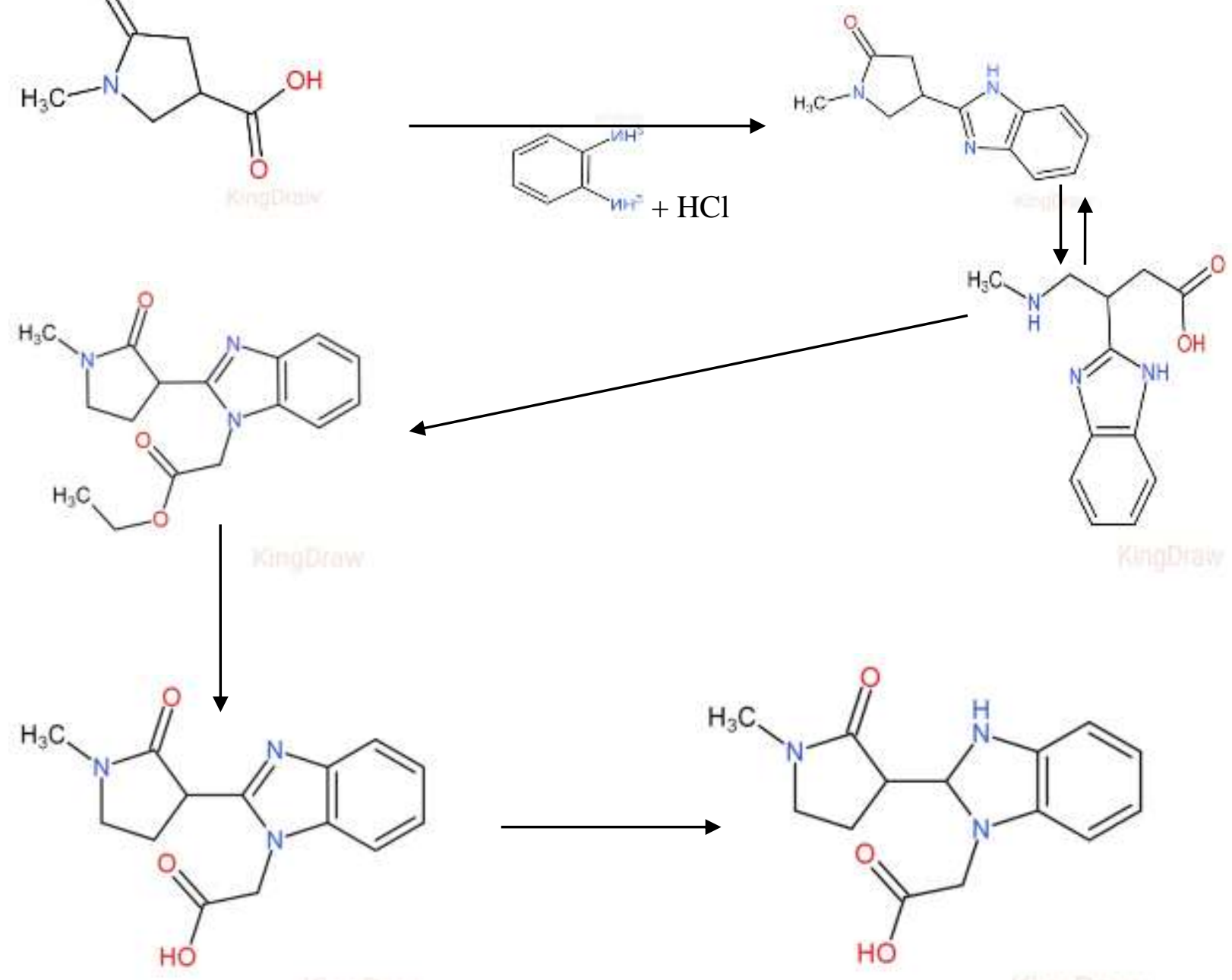

\section{Biological Profiles:}

\section{Antiprotozoal activity:}

A series of 2-(trifluromethyl)-H-benzimidazole derivatives (4a-4i) synthesized by Lillian Y. Mulia et al. The compound 4a-4i was evaluated in-vitro antiprotozoal activity against G. intestinalis E. histolytica T. vaginalis and L. Mexicana. Compound 4b, 4c and 4e showed most potent activity. The order of parasite susceptibility found was E. histolytic $>$ G. intestinalis $>$ T. vaginalis > L. Mexicana.

\section{Anticoagulant:}

A series of halothiophene benzimidazoles as p1 surrogates of inhibitors of blood coagulation factor Xa was synthesized by Werner W. K. R. Mederski et al. All synthesized compounds were assayed against human $\mathrm{f} \mathrm{Xa}$, thrombin and trypsin. The compound $5 \mathrm{a}$ and $5 \mathrm{~b}$ showed potent activity.

\section{Antifungal activity:}


Synthesis of phenyl hydrazine substituted benzimidazole derivatives and their biological activity by Tiwari A. et al. The 6-nitroderivative of benzimidazole shows good activity against Aspergillus Niger and Aspergillus flavus.

\section{Antihistaminic:}

A series of substituted (3- phenoxypropyl )amines were reported by Robert aslanian et al. All the synthesized compounds evaluated for antihistamine activity. Compound 13a showed potent H1 antagonist possessing a good pharmacokinetic profile in the rat.

\section{Antiulcer activity:}

A series of novel pyrimidylthiomethyl benzimidazole were reported by Bariwal J. B. et al. All synthesized compounds evaluated for the antiulcer activity by pylorus ligation of rats. Compounds (11a) and (11b) when evaluated significantly decreased the gastric acid secretion, free acidity as well as gastric ulcer in the pylorus ligated rats and the effects are dose dependent and comparable to omparazol. The compound $11 \mathrm{~b}$ was more effective than $11 \mathrm{a} .{ }^{[8]}$

\section{DERIVATIVES OF BENZIMIDAZOLE:}

\subsection{1, 2, and 4- triazalo [2, 3-a] benzimidazole-2-thione:}

\section{Structure:}

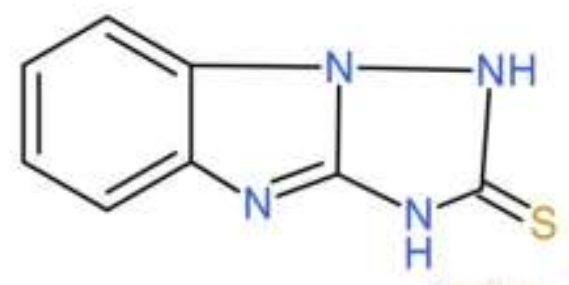

\section{Synthesis:}

To a stirred solution of 1, 2 diaminobenzimidazole (10.0g, $0.052 \mathrm{~mol})$ in DMF (100 ml), CS2 (30 $\mathrm{ml}, 0.47 \mathrm{~mol}$ ) was added. The reaction mixture was refluxed for $16 \mathrm{~h}$ and formed precipitate was filtered, washed with methanol and dried. The product is insoluble in most organic solvent; hence it was purified by dissolving in $5 \% \mathrm{KOH}$. The alkaline solution was cooled in an ice bath, and then rendered acidic by addition of conc. HCL under stirring. The process of purification was repeated until constant melting point. ${ }^{[9]}$

\section{Anti-inflammatory and analgesic activity:}

Animals were housed in separate cages 6 animals each in temperature-controlled rooms at $25^{0} \mathrm{c}$. Animals were allowed free access to food and water and maintained at a $12 \mathrm{~h} \mathrm{light} \mathrm{/} \mathrm{dark} \mathrm{cycle.}$ Work was conducted in a accordance with the internationally accepted principles for laboratory animals use and care as found in the European community guidelines. 


\section{1, 2- Di-substituted Benzimidazole:}

\section{Structure:}

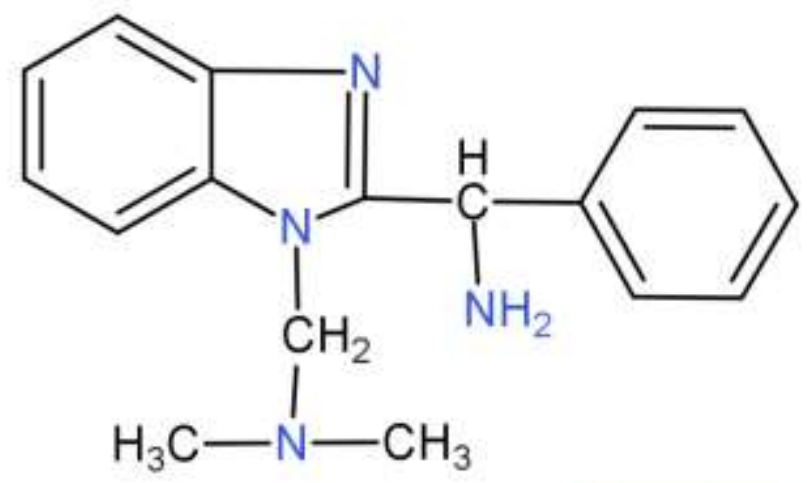

\section{Synthesis:}

Benzimidazole was synthesized by $\mathrm{O}$ - phenylene diamine $(12 \mathrm{mmol})$ and phenyl glycine (36mmol) were stirred in $4 \mathrm{~N} \mathrm{(} 40 \mathrm{ml}$ ) and refluxed for 4 hour, then cooled at room temperature. The completion of this reaction was monitored by thin layer chromatography. The resulting brown solid was filtered and wash with water dried in vacuum and recrystallized from acetone.

The above mentioned procedure for the synthesis of benzimidazole derivatives using conventional various carboxylic acid in presence of conc. HCL, but in presence work we have use mannish bases, which gives advantage for various substitution in benzimidazole nucleus. ${ }^{[10]}$

\section{2- ethylbenzimidazole :}

\section{Structure:}

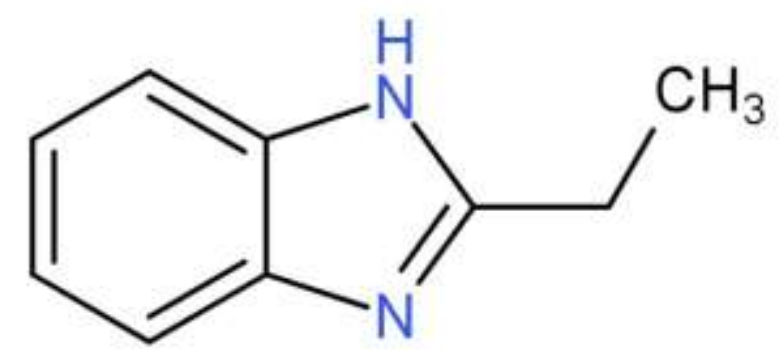

\section{Synthesis:}

A mixture of $\mathrm{O}$ - phenylenediamine ( 0.01 mole $)$ and propionic acid (0.01 mole ) was refluxed for $3 \mathrm{hrs}$ in $4 \mathrm{~N}$ hydrochloric acid ( $20 \mathrm{ml}$ ) on a water bath. The reaction mixture was cooled and basified with ammonium hydroxide solution to obtain a precipitate. The precipitate was air dried and purified by recrystalization from $50 \%$ ethanol. The product was obtained as a colourless crystaline solid m.p. $172{ }^{\circ} \mathrm{c}$ and yield $71 \% .^{[11]}$ 


\section{Thiabendazole:}

\section{Structure:}

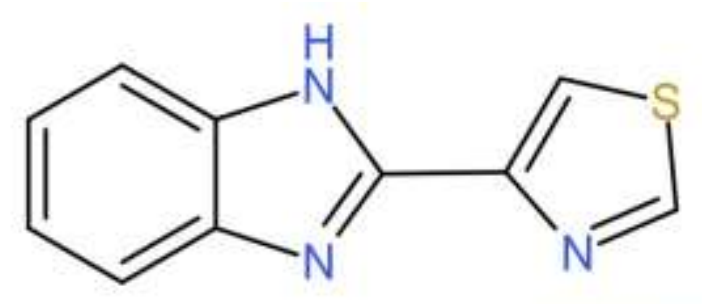

\section{Synthesis:}

1.Thiabenzadol is produced by heating thiazoly-2-formamide with o-phenylenediamine in the presence of polyphospheric acid.

2.Preparation by reaction of 4-thiazolecarboxamide with o-phenylenediamaine in polyphospheric acid

\section{Uses:}

1. fungicide

2. controll of aspergillus, botrytis, cercospora ,septoria, etc.

3. for the treatment of hookworms

\section{Synthesis of [1-(N-subtituted amino)methyl]-2-ethyl benzimidazole :}

\section{Structure :}

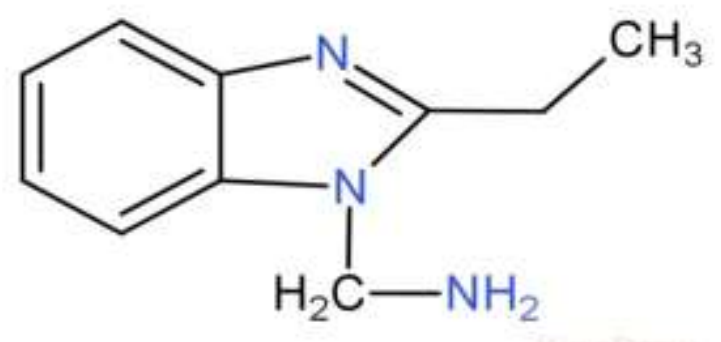

\section{Synthesis:}

2-ethyl benzimidazole in methanol was stirred at RT with parafrmaldehyde conc. Hydrochloric acid and appropriate primarylsecondary amine for 1 hour. It was refluxed for 2 hour, filterd in hot condition and filterate and the filterate was conc. To one third of its original volume. The residual liquid was left in refrigerator for $24 \mathrm{hrs}$. The product that seprated out was purified ether by columnchromatography or by recrystalization to get a pure crystaline mannich base. ${ }^{[13]}$

\section{6. (1H-benzimidazol-2ylmethyl)-(3,4-dichloro-phenyl)-amine:}

Structure : 


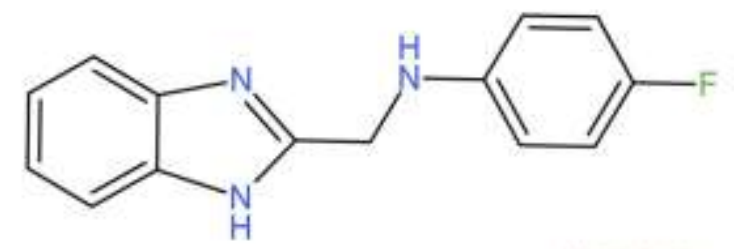

\section{Synthesis:}

A mixture of o-phenylenediamine and monochloroacetic acid was refluxed for $3 \mathrm{~h}$ in $4 \mathrm{~N}$ hydrochloric acid on a water bath. The reacton mixtured was cooled and basified with ammonium hydroxide solution. The precipitate thus obtain dried and recrystralised from methanol with activated charcoal treatment. The pure product obtained was slightly yellow coloured crystal. ${ }^{[14]}$

\section{2-(4-bromophenyl)-1H-benzimidazol :}

\section{Structure:}

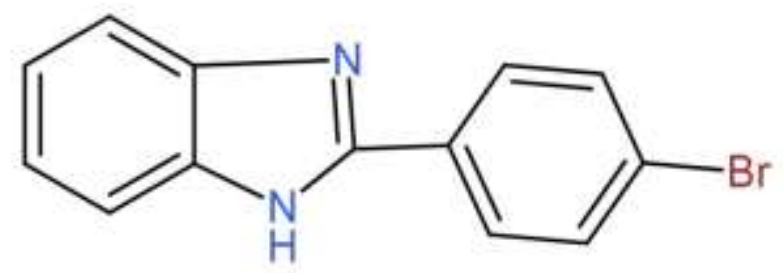

\section{Synthesis :}

A mixture of o-phenylenediamine, 4-bromo benzoic acid in presence of polyphosphoric acid was heated $180^{\circ} \mathrm{c}$ for 4 hours. After confirmation of completion of the reaction, the mixtured was chilled in ice and nutralized with $40 \%$ aqueous sodium hydroxide solution to $\mathrm{pH} 10$. The obtain crude material which was recrystalized in ethanol water to obtain pure product. ${ }^{[15]}$

\section{2-(4-bromophenyl)-1H-benzimidazole :}

\section{Structure :}

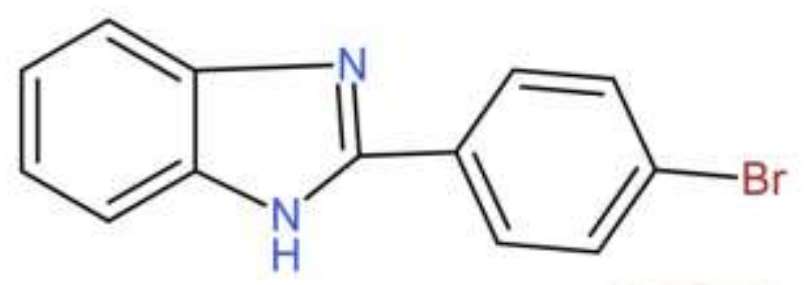

\section{Synthesis :}

To a solution of 2-(4-bromophenyl)-1H-benzimidazole and potassium carbonate in acetone was added compound at RT. The reaction mixture was heated to reflux for 3 to 4 hours. Reaction 
progress was monitered on TLC. After reaction completion distilled out solvent under reduce pressure at 40 to $50{ }^{\circ} \mathrm{c}$ and added $30 \mathrm{ml}$ water and $30 \mathrm{ml}$ ethyl acetate and aqueous layers were seprated. Ethyl acetate layer were seprated. Ethyl acetate layer was washed $30 \mathrm{ml}$ water. Distilled out ethyl acetate under reduce pressure to get a digass mass and which was recrystalization in ethanol and water to obtained corresponding $\mathrm{N}$-subtituted derivative. ${ }^{[16]}$

\section{CONCLUSION:}

Modification on benzimidazole moiety displayed valuable biological activities. The benzimidazole ring is an important pharmacophore in modern drug discovery. Benzimidazole derivative are resource for medicinal research.

\section{REFERENCES:}

1. Rishabh D. Pandey et al; "synthesis characterization and pharmacological screening of novel benzimidazole derivatives. Arabian Journal of Chemistry 10.1016/j.arabjc.2011.04.013

2. https://en.wikipedia.org/wiki/Benzimidazole

3. Patil A, Ganguly S and Surana S. A systematic review of benzimidazole derivative as an antiulcer agent. Rasayan J Chem. 2008; 1(3):447-460

4. Kubo K, Oda K, Kaneko T ,Santosh H and Nohara A. synthesis of 2-(4-fluoroalkoxy-2pyridyl ) methyl [sulfinyl]-1H-benzimidazoles as antiulcer agents. Chem Pharma Bull. 1990; 38(10):2853-2858.

5. Uchida M, Chihiro M, Morita S, Yamashita H, Yamasaki K, Kanbe T ,Yubuuchi Y, and Nakagawz K. synthesis and antiulcer activity of 4- substituted 8-[(2-Benzimidazoyl )sulfenylmethyl]-1,2,3, 4-tetrahydroquinolines and related compounds .Chem Pharm Bull. 1990; 38(6):1575-1586.

6. Grassy A, Ippen J , Bruno M, Thomas G and Bay P. A Thiazolyamino benzimidazole derivative with gastroprotective properties in the rat. Eur J. Pharmacol. 1991; 195(2):2519.

7. Ozkay Y, Tunali Y, Karaca H, Iskidag I. Antimicrobial activity and a SAR study of some novel benzimidazole derivatives bearing hydrazone moiety. European Journal of Medicinal Chemistry.2010; 45(8):3293-3298.

8. Benzimidazole: AS Potential Biologically Active Agent Vikash Kumar Chaudhari, Devender Pathak, Satyendra Singh Department of Pharmaceutical Chemistry, Rajiv Academy for Pharmacy, Mathura, India 
9. Mohamed BG, Abdel-Alim M and Hussein MA. Synthesis of 1-acyl-2-alkylthio-1, 2, 4 triazolobenzimidazoles with antifungal, anti-inflammatory and analgesic effects. Acta Pharm 2006; 56: 31-48.

10. Reddy BA. Synthesis, Characterization and Biological Evaluation of 1, 2- disubstituted benzimidazole derivatives using Mannish Bases. E-J. Chem 2010; 7, Supply 1: 222-226.

11. Mariappan G, Bhuyan NR, Kumar P, Kumar D and Murali K. Synthesis and biological evaluation of Mannish bases of benzimidazole derivatives. Indian J. Chem 2011; 50Suppl B: 1216-1219

12. .https://pubchem.ncbi.nlm.nih.gov/compound/Thiabendazole\#section=Uses

13. Kumar B V and Reddy V M , Indian drugs ,23(2),1985, 98.

14. Synthesis and biological evaluation of 2-substituted benzimidazole derivatives Gurusamy Mariappan, Rajiv Hazarika, Faruk Alam, Rashmi Karki, Uddhav Patangia, Shyamalendu Nath Dept. of Medicinal Chemistry, Himalayan Pharmacy Institute, Majhitar, EastSikkim 737 136, India.

15. Synthesis, Characterization and Biological Evaluation of Novel $\mathrm{N}$ alkylated 2-(4 bromophenyl)-1H-benzimidazole Derivatives Deshmukh SK, Sanjay Dashrath Vaidya. Department of Chemistry, Jagdishprasad Jhabarmal Tibrewala University, Jhunjhunu, Rajasthan, INDIA. 